\title{
Non-Steady Evolution of the Accretion Disk Solar Nebula
}

\author{
Hiroshi MIzUnO ${ }^{1}$ and Kiyoshi NAKAZAwa ${ }^{2}$ \\ ${ }^{1}$ Notre Dame Seishin University, Okayama 700, Japan \\ ${ }^{2}$ Department of Applied Physics, Tokyo Institute of Technology, Tokyo 152, Japan
}

(Received June 17, 1994; Revised July 14, 1994; Accepted July 14, 1994)

\begin{abstract}
We have studied the non-steady evolution of both gas and solid grains in the convective solar nebula by using accretion disk model. Time-dependent processes considered are the accretion from the outer boundary of the nebula, the collisional growth of grains, the sublimation/recondensation of solid ice.

The calculations show that the grain growth weakens the convection, and therefore the laminar nebula is left. The remaining nebula has about 0.05 solar mass with the lifetime of about $10^{7}$ years. This remnant mass shows weak dependences on the efficiency factor $\alpha$ in the turbulent viscosity and the accretion rate. The spatial distribution of the remnant gas is determined by the size of grains because they grow to the size of $\mathrm{mm}-\mathrm{cm}$. This remnant mass is smaller than that of Ruden and Pollack (1991) because of the heatings besides the dissipation which decreases at the final stage. Thus the grains and the heating sources are the keys to guess the remaining mass and its distribution, or in other words, the initial condition of planetary accumulation.
\end{abstract}

\section{Introduction}

The aim of this paper is to study the time-dependent evolution of the primitive solar nebula from a viewpoint of accretion disk model and to see the role of solid grains on the evolution. This study is initiated by observational developments of young stellar objects and by theoretical progresses of interpretations of the new discoveries. For example, the observed infrared excess for $\mathrm{T}$ Tauri stars can be probably explained as the radiation from thin disks around the young stars (Kenyon and Hartmann, 1987; Adams et al., 1988; see also the review by Bertout (1989)). Hydrodynamical calculations of collapse of molecular cloud core show how these disks as well as central stars are built (see a review by Tscharnuter (1991) for recent developments and references). A gravitationally unstable molecular cloud core begin to collapse in a free-fall time scale. Because of rotation of the cloud, not all of the cloud accumulates directly onto a central stellar embryo but forms a flattened disk around it with the aid of turbulent viscosity. The subsequent evolution of the star/disk system is regulated by the outward transport of angular momentum in the disk. During and after the infall stage, the disk mass is considered to accrete onto the central star by the action of local viscosity or global instability with a time scale probably longer than the infall time. Thus the central star further grows by radial accretion through the disk. We apply this model to the solar nebula and the protosun.

There are many studies on the evolution of the solar nebula. Safronov (1972) and Hayashi et al. (1985) assumed a so-called minimum solar nebula to form planets by accumulation. Their quiet nebulae give the initial condition of planetary accumulation, not considered from the evolutionary aspect. On the other hand, Cameron (1978) was lead to the giant protoplanet hypothesis by investigating the evolution of a massive nebula. Lin and Papaloizou $(1980,1985)$ showed that if the opacity depends on temperature strongly enough as the grain opacity does in the Rayleight limit, the nebula becomes convectively unstable. They also studied the time variation of the nebula by using an $\alpha$-model of turbulent viscosity with the efficiency factor $\alpha$ fixed in space and 
time. Cabot et al. (1987a) found the efficiency factor $\alpha$ to be much smaller in the geometrically thin nebula than expected in spherical stellar interior, and applied the result to the solar nebula on the assumption of steady mass flux in the nebula (1987b). Lin and Pringle (1990) investigated the evolution of a gravitationally unstable nebula by arguing that gravitational instability creates viscosity alternative to the convective viscosity.

Ruden and Pollack (1991, hereafter RP) recently elaborated the non-steady evolution of protostellar disks in which the viscous heating balances with the radiative loss. They argued that the optical depth, calculated with the opacity for interstellar grains, determines the efficiency factor $\alpha$ of thermal convection. Then $\alpha$ depends on time and position. According to their treatment of $\alpha$, the depletion of the disk mass due to the accretion onto the central star lowers the optical depth and hence weakens the convection, resulting in the suppress of the radial migration of the disk mass. They concluded that the disk of about 0.1 solar mass is left within several $\times 10^{5}$ years after the infall stage ends.

The solid component in the nebula is expected to affect the evolution of the nebula. The early works paid attention to the grains' role as materials of planets (Goldreich and Ward, 1973; Nakagawa et al., 1981; Weidenschilling, 1984). From the calculation of growth and shattering of grains in the steady turbulent nebula, Weidenschiling suggested the importance of optical property and its change as the grains evolve, which was also indicated by Mizuno et al. (1988) in the presence of interstellar grains supplied by the accreting cloud gas. Corresponding to the low estimate of $\alpha$ by Canuto et al., Battaglia (1987) re-studied the growth calculation of grains. Mizuno (1989) calculated the grain growth including the radial migration in the steady turbulent nebula following the formulation by Morfill and Völk (1984). The behavior of grains and its vapor in turbulent nebula was studied from cosmochemical aspects by Cameron and Fegley (1982) and by Morfill (1983). For a low $\alpha$, Morfill (1988) gave the nebula structure with steady mass flux and constant $\alpha$ in the consideration of grain opacity change due to growth and sublimation.

From the above review, we find the lack of study which takes account of time-dependent evolutions of both nebula and solid grains in a consistent way. In reality, the evolving nebula changes both spatial and size distributions of grains. The change in optical property, caused by the evolving grains, in turn affects the evolution of the nebula. In this paper we will pursue this problem after the infall of cloud gas finishes building the nebula disk. We treat only the inner region of the disk inside the radius of $40 \mathrm{AU}$, that is, the scale of the present solar system. The nebula outside this radius is assumed to be replaced by the mass flux at $40 \mathrm{AU}$. (We call this radial mass influx through the boundary the radial accretion or simply the accretion hereafter.) Another reason to restrict ourselves only in the inner region is that magnetic field may play an important role in the outer region because cosmic ray particles penetrate the nebula gas to ionize it in the less dense region (Hayashi, 1981). The magnetic effect, quite sensitive to the distance from the protosun, is difficult to evaluate quantitatively. In this way we formulate the evolution of the solar nebula as an accretion disk model with the accretion rate $\dot{M}(t)$ at $40 \mathrm{AU}$, which would decrease with finite time scale because the inward drift flow depletes the outer disk.

The radial drift flow within the nebula is most plausibly induced as reactions to viscous angular momentum transfer due to turbulence. We assume thermal convection as a transfer mechanism of angular momentum because it is extensively examined and well understood. Considering the diffusion time comparable with the expected evolutional time of the system, the radial flow in the disk and other quantitites become time-dependent. This tendency is caused also by other processes involved in the system: the varying accretion rate, the opacity change due to the varying size and spatial distribution of grains and the increasing mass of the protosun. Therefore it is worthwhile to study the non-steady evolution of the solar nebula and the protosun.

We summarize the ingredients. We use the estimation of turbulent velocity by Cabot et al. $(1987 \mathrm{a})$, that is, the convective velocity about $10^{-4}-10^{-2}$ times as small as the local sound velocity for the geometrically thin nebula. This estimation is used in an approximate way as in 
Eq. (1) in Section 2, similar to that used by RP. The effective viscosity, determined by Eq. (2) below, regulates the transfer rate of angular momentum and therefore the drift velocity. It also dissipates the turbulent energy. These will be considered in Eqs. (3) and (6).

According to RP, the optical depth is a key factor for the evolution of the protostellar disk. We consider the following process which vary the size and space distributions of grains: coagulation, sublimination/recondensation, orbital decay and turbulent diffusion. Concerning with the geometry of grains, we assume the spherical grains for simplicity. Including all these processes enables us to evaluate the opacity in the whole range of grain size. By the way it is noted that the detailed structure and geometry of grains should be important factors for the evolution of the solar nebula because they determine the aerodynamical and optical properties of grains (Meakin and Donn, 1988). The evolutional studies from this standpoint should be done in the future with deeper understandings of grains' nature in the solar nebula.

One of the important external parameters is the accretion rate, that is, the inward flux at $40 \mathrm{AU}$, which would be determined probably by the magnetic viscosity in the outer disk. In our treatment this parameter is left as a free parameter from the reason stated above. However the accretion continues only during a finite time scale. It at least weakens as the outer disk depletes. Therefore whether or not the gas flow in the inner disk is steady depends on the accretion time and the viscous diffusion time. Therefore we treat both gas and solid grains in a full non-steady way.

With these remarks in mind, the evolution of the nebula will be formulated in Section 2 . The assumptions and simplifications will be also mentioned. In Section 3 the numerical results will be presented, and the conclusion and comments will be summarized in Section 4 .

\section{Assumptions and Basic Equations}

\subsection{Assumptions on convection}

First of all we mention major assumptions on turbulence. In order to handle the turbulence precisely, the convective velocity should be evaluated by solving the vertical structure of the nebula and applying a stability condition. In this study, however, we do not follow this strict way for the purpose of saving computational time. Instead we assume a uniform vertical distribution of all quantities over the half-thickness. Furthermore the convective velocity $v_{t}$ is assumed to be expressed by the following equation, i.e.,

$$
v_{t}=\alpha c_{s} \frac{\tau-1}{\tau+1}
$$

where $c_{s}$ is the sound velocity. With the opacity $\kappa$ and the surface density $\Sigma$, the optical thickness $\tau$ is given by $\kappa \Sigma / 2$. If $\tau$ is large enough, Eq. (1) reduces to the result of Cabot et al. (1987a) with $\alpha$ between about $10^{-4}$ and $10^{-2}$. If the grain opacity is small and the convection weakens, $v_{t}$ decreases owing to the last factor in Eq. (1) (as seen in Section 3, $\tau$ is greater than unity in our calculations). We fix $\alpha$ to be $3 \times 10^{-3}$ for simplicity. Thus we expect Eq. (1) to approximate the real situation. Note that Eq. (1) is similar to the approximation of RP if we put $n=1$ and $\tau_{\text {crit }}=2$ in Eq. (11) of their paper. The effective viscosity $\nu$ is assumed to be given on an analogy of gas kinematics:

$$
\nu=\frac{1}{3} v_{t} h
$$

where $h$ is the half-thickness of the nebula.

\subsection{Equations for gaseous component}

We consider, under the following assumptions, the gaseous component of the nebula which evolves under the action of viscosity. By neglecting the self-gravity and the radial pressure 
gradient, the gas is assumed to revolve with the Keplerian angular velocity $\Omega=\left(G M_{c} / r^{3}\right)^{1 / 2}$, where $M_{c}$ is the mass of the protosun, $r$ the distance from the protosun, and $G$ the gravitational constant. Then the half-thickness $h$ is given by $c_{s} / \gamma^{1 / 2} \Omega$ where $\gamma$ is the ratio of specific heats. Integrations of the equations of motion and continuity over the vertical direction give, respectively,

$$
\frac{\partial}{\partial t}(\Sigma j)+\frac{1}{r} \frac{\partial}{\partial r}\left(r \Sigma j v_{r}\right)=\frac{1}{r} \frac{\partial}{\partial r}\left\{\Sigma \nu r^{3} \frac{\partial}{\partial r}\left(\frac{j}{r^{2}}\right)\right\}
$$

and

$$
\frac{\partial}{\partial t} \Sigma+\frac{1}{r} \frac{\partial}{\partial r}\left(r \Sigma v_{r}\right)=0
$$

in cylindrical coordinates and with time $t$. The radial drift velocity is denoted by $v_{r}$ and $j=r^{2} \Omega$. From Eqs. (3) and (4), $v_{r}$ is given by

$$
v_{r}=-\frac{3}{\Sigma r^{1 / 2}} \frac{\partial}{\partial r}\left(\Sigma \nu r^{1 / 2}\right)-r \frac{\dot{M}_{c}}{M_{c}}
$$

where $\dot{M}_{c}$ is the time derivative of $M_{c}$ (Safronov and Ruzmaikina, 1985). Inserting Eq. (5) into Eq. (4), we have a diffusion equation for $\Sigma$.

The equation of energy supplements the above equations. By assuming $v_{r}, c_{s} \ll r \Omega$ and taking account of possible processes of heating and cooling, it becomes

$$
\begin{aligned}
& \frac{\partial}{\partial t}\left(\Sigma \frac{1}{\gamma-1} \frac{k T}{\mu H}\right)+\frac{1}{r} \frac{\partial}{\partial r}\left(r \Sigma v_{r} \frac{\gamma}{\gamma-1} \frac{k T}{\mu H}\right) \\
& \quad=\frac{9}{4} \Sigma \nu \Omega^{2}+\frac{L h q}{4 \pi r^{3}}+2 \sigma T_{o}^{4}-\frac{16 \sigma T^{4}}{3 \kappa \Sigma}+\frac{2}{r} \frac{\partial}{\partial r}\left\{h r \frac{4 a c T^{3}}{3 \kappa \rho} \frac{\partial T}{\partial r}\right\}
\end{aligned}
$$

where $\mu$ is the mean molecular weight, $H$ the mass of a hydrogen atom, $k$ the Boltzmann constant, $\sigma$ the Stephen-Boltzmann constant, and $L$ the luminosity of the protosun. Moreover $T$ and $\rho(=\Sigma / 2 h)$ are the temperature and the density of gas, respectively. The terms in the right hand side correspond to the following processes - the first term: heating due to viscous dissipation, the second: heating due to the solar radiation incident from the surfaces of the nebula, the third: heating due to cosmic ray, the fourth: cooling due to vertical radiation loss, and the fifth: radial transfer of radiation. In the third term the heating rate by cosmic ray is replaced by black body radiation of $T_{o}=10 \mathrm{~K}$ which is the approximate value obtained with the space energy density and velocity of cosmic rays (Allen, 1973). The radial transfer of radiation is considered to be small if the opacity changes smoothly in the radial direction. Nevertheless we retain this effect as the fifth term in order to respond the possible sharp change of opacity due to ice sublimination and grain growth. Another reason to have the fifth term in the energy equation is to take account of the head-on input of solar irradiation from the inner boundary and to keep the temperature at the outer boundary at the fixed temperature. Note that the factor $q(=d \ln h / d \ln r-1)$ in the second term arises from the incident angle of the solar radiation (note that we assume $h / r \ll 1$ in deriving the second term). In order to avoid the numerical instability included in the second term, we fix $q$ to be 0.2 (see, for example, Hayashi et al. (1985)). The calculations show that $q$ obtained from the nebula model is in good agreement within a factor of two except the outermost region. Note that RP considered only the first and fourth terms.

\subsection{Equations for solid component and its vapor}

We treat the mass density of chemical species of solid, their vapor and the number density of solid grains on the basis of their formalism of Morfill and Völk (1984), reducing the number of species to a small number of typical species: solid grains (composed of ice and rock/metal), solid 
ice, ice vapor and number of solid grains. The followings are the equations for the surface density of grains $\Sigma_{d}$, that of solid ice $\Sigma_{i}$, that of ice vapor $\Sigma_{v}$, and the surface number density $N$. We consider only grains with mean mass $m$ defined by $\Sigma_{d} / N$ in order to avoid the complexity and long CPU time for computing the grains' mass spectrum.

$$
\begin{gathered}
\frac{\partial}{\partial t} \Sigma_{d}+\frac{1}{r} \frac{\partial}{\partial r}\left[r\left\{\Sigma_{d} v_{r d}-\Sigma \nu_{d} \frac{\partial}{\partial r}\left(\frac{\Sigma_{d}}{\Sigma}\right)\right\}\right]=N \dot{m}_{r s} \\
\frac{\partial}{\partial t} \Sigma_{i}+\frac{1}{r} \frac{\partial}{\partial r}\left[\frac{\Sigma_{i}}{\Sigma_{d}} r\left\{\Sigma_{d} v_{r d}-\Sigma \nu_{d} \frac{\partial}{\partial r}\left(\frac{\Sigma_{d}}{\Sigma}\right)\right\}\right]=N \dot{m}_{r s} \\
\frac{\partial}{\partial t} \Sigma_{v}+\frac{1}{r} \frac{\partial}{\partial r}\left[r\left\{\Sigma_{v} v_{r}-\Sigma \nu \frac{\partial}{\partial r}\left(\frac{\Sigma_{v}}{\Sigma}\right)\right\}\right]=-N \dot{m}_{r s}
\end{gathered}
$$

and

$$
\frac{\partial}{\partial t} N+\frac{1}{r} \frac{\partial}{\partial r}\left[r\left\{N v_{r d}-\Sigma \nu_{d} \frac{\partial}{\partial r}\left(\frac{N}{\Sigma}\right)\right\}\right]=-\frac{1}{2} \sigma_{c} v_{c} \frac{N^{2}}{2 h}
$$

where $v_{r d}$ is the radial drift velocity, $\nu_{d}$ the diffusion coefficient, $\sigma_{c}$ the collision cross section (= $=4 \pi a^{2}$ : $a$ being a mean grain radius), $v_{c}$ the collision velocity, and $\dot{m}_{r s}$ the grain's mass increase per unit time due to recondensation/sublimation of ice: these are all for grains. The mass flux of solid ice in Eq. (8) is obtained from its abundance relative to the whole grain as well as the total mass flux of grains in Eq. (7), by assuming each grain to be a mixture of solid ice and rock/metal. We also assume each grain to have the fixed internal density $\rho_{s}\left(=2 \mathrm{~g} / \mathrm{cm}^{3}\right)$ in spite of the varying ratio of solid ice to rock/metal, for simplicity. Note that $\Sigma_{v}$ is coupled through $\dot{m}_{r s}$ as seen from Eq. (11) below.

The expressions for $v_{r d}$ and $\nu_{d}$ should be referred to Mizuno (1989). Moreover $\dot{m}_{r s}$ is given by

$$
\dot{m}_{r s}=\pi a^{2}\left\{c_{m i} \frac{\Sigma_{v}}{2 h}-\frac{8}{\pi} \frac{P_{s}(T)}{c_{m i}}\right\}
$$

where $P_{s}$ is the saturated pressure of ice vapor and $c_{m i}$ is the mean thermal velocity given by $\left(8 k T / \pi \mu_{d} H\right)^{1 / 2}\left(\mu_{d}\right.$ being the mean molecular weight of ice vapor $\left.(=18)\right)$. As to $v_{c}$, we adopt an approximate formula by Völk et al. (1980), although there remains uncertainty in this formula as pointed out by Weidenschilling (1984). See Subsection 3.4 about this point.

As seen in the collision term in Eq. (10), we simplify the collisional outcome of spherical grains as coagulation without shattering or erosion. The shattering occurs for larger colliding grains owing to the larger turbulent collision velocity. In the fully-developed turbulence, the shattering suppresses the grain growth beyond about $\mathrm{cm}$-size (Weidenschilling, 1984). In a turbulent nebula with lower turbulent velocity estimated by Cabot et al. (1987a), the shattering is less effective, allowing to create grains of sizes up to a few tens of centimeters (Battaglia, 1987). Therefore as long as the size is concerned, the shattering would not change our results because the maximum size of mean grains is about $\mathrm{cm}$ in the presence of radial drift (see Subsection 3.2).

For moderate collision velocity, a part of grains is excavated on collision and returned to the grains of smaller sizes. Let us evaluate how this erosion affects our simple treatment with mean grains in calculating mass density and grain opactity. To do so, we approximate the mass spectrum with double peaks: the peak at grain mass $M$ (mass density $\rho(M)$ ) corresponding to the mean grain mass which grows by collision, and the peak at grain mass $m_{0}$ of radius $1 \mu \mathrm{m}$ (mass density $\rho\left(m_{0}\right)$ ) corresponding to the grains returned by erosion. Then $\rho\left(m_{0}\right)$ is determined by the production rate due to erosion on the collisions between the grains $M$, and the growth rate by the collisions between the grains $m_{0}$ and the collisions between the grains $m_{0}$ and the 
grains $M$. This condition gives us the mass density ratio as discussed in Appendix. For example, when $T=200 \mathrm{~K}, \Sigma=1000 \mathrm{~g} / \mathrm{cm}^{2}$ and $E_{s}$ (strength parameter of grains) $=5 \times 10^{4} \mathrm{erg} / \mathrm{cm}^{3}$, $\rho\left(m_{0}\right) / \rho(M) \sim 2 \times 10^{-7}\left(\alpha / 10^{-3}\right)^{3}$ for $10^{-4}<\alpha<10^{-2}$ and $a_{M}=1 \mathrm{~cm}$, where $a_{M}$ is the radius of grain $M$ and the value of $E_{s}$ is taken from Weidenschilling (1984) and Battaglia (1987). Thus we can ignore the mass density of eroded grains compared with that of growing grains.

With this result, we further estimate the opacity due to the grains $m_{0}$. If the size of the growing grains $M$ is less than the wavelength $\lambda$, the erosion has no effect on the opacity. However, when the size of the grains $M$ exceeds $\lambda$, the grains $m_{0}$ has a possibility to be an appreciable source of opacity relative to that of the grains $M$ even though $\rho\left(m_{0}\right) / \rho(M) \ll 1$ because the opacity due to $M$ decreases as it grows. According to the discussion in Appendix, the ratio of the opacity $\kappa\left(m_{0}\right)$ due to grain $m_{0}$ to the opacity $\kappa(M)$ due to grain $M$ is given by $\kappa\left(m_{0}\right) / \kappa(M) \sim$ $1 \times 10^{-4}\left(\alpha / 10^{-3}\right)^{3}$ for the same range of $\alpha$ and conditions as the previous paragraph. Thus in our case of $\alpha=10^{-4}$ to $10^{-2}$, the relation $\kappa\left(m_{0}\right) / \kappa(M)<$ the order of 0.1 holds and the grains $m_{0}$ is able to be neglected in opacity. These results indicate that the erosion as well as the shattering has little effect on our calculation of grain size and grain opacity in average if $\alpha$ is as small as expected by Cabot et al. (1987).

Since the recondensation/sublimation progresses faster than the radial diffusion if it happens, ice vapor is assumed to be in equilibrium with solid ice, that is, $\dot{m}_{r s}=0$. Then the numerical integration to proceed one time step is divided into two stages. At first, the temporal $\Sigma_{d}, \Sigma_{i}$, and $\Sigma_{v}$ are obtained from Eqs. (7) to (9) with $\dot{m}_{r s}=0$, including only the effects of drift and diffusion. Next, the equilibrium condition determines the redistribution between solid ice and ice vapor under the constraint that their total amount is preserved, which gives the new variables for the next time step.

\subsection{Boundary conditions}

Since we do not know exactly what happens in the innermost boundary region between the nebula and the protosun where the magnetic field should be important, the inner boundary conditions are imposed in a simplified manner. That is, all of $\Sigma, \Sigma_{d}, \Sigma_{i}$, and $\Sigma_{v}$, and $N$ vanish at the inner edge of the nebula $r=R_{i}$, implicitly assuming that some efficient mechanisms immediately remove the nebula gas, grains and ice vapor which are flowing out of the inner boundary toward the protosun. The temperature needs to be satisfied with

$$
-\left.\frac{4 a c T^{3}}{3 \kappa \rho} \frac{\partial T}{\partial r}\right|_{r=R_{i}}=\frac{L}{4 \pi R_{i}^{2}}-\sigma T_{i}^{4}
$$

where $T_{i}$ is the temperature at $r=R_{i}$. Note that our inner boundary conditions for solid components differ from those of Morfill and Völk (1984). They imposed these conditions at the "sublimation boundary" where solid species were assumed to evaporate instantly. In our treatment, the evaporation occurs gradually in the wide region (see Section 3), coupling with the gentle temperature variation there. Thus we cannot define the sublimation boundary and instead impose the inner boundary condition at the inner boundary of the disk.

The outer boundary condition is rather simple. Assuming the solar abundance and all icy component in solid for the accreting cloud gas, the mass flux for gas is given by $-\dot{M}(t)$ at the outer boundary $r=R$, that for grains by $-A \dot{M}(t)$, that for solid ice by $-A_{\mathrm{H}_{2} \mathrm{O}} \dot{M}(t)$, that for ice vapor by zero, and the number flux for grains by $-A \dot{M}(t) / m_{0}$. Here $\dot{M}(t)$ is the accretion rate through the outer edge of the nebula, $A$ the solar abundance of heavy element relative to $\mathrm{H}$ and $\mathrm{He}(=0.016), A_{\mathrm{H}_{2} \mathrm{O}}$ that of water $(=0.013)$, and $m_{0}$ the mass of an interstellar grain of 1 $\mu \mathrm{m}$ in radius. The temperature is equated to $T_{o}$ at $r=R$, determined by cosmic ray.

As a supplementary condition, an equation is necessary for describing the growth of the 
protosun. That is,

$$
\frac{d M_{c}}{d t}=-\left.2 \pi r \Sigma v_{r}\right|_{r=R_{i}}
$$

\subsection{Opacity}

The grain opacity has different properties depending on grain radius $a$. In the Rayleigh limit regime where $a \ll \lambda$ (the characteristic wavelength of local thermal radiation), the opacity is proportional to the grain abundance relative to gas and independent of $a$. This opacity is well studied for the gas with the solar abundance (Pollack et al., 1985, RP). In this study we use an approximate formula for Rayleigh limit made as follows. First we adopt the expression from Pollack et al. (1985) for solar abundance. Then we correct it so as to allow the deviation of the grain abundance from the solar one. This is because the solar abundance is not always guaranteed in our case owing to different drift velocities and diffusion coefficients between gas and grain. In another limit where $a \gg \lambda$, the opacity is well approximated by twice of the total geometrical cross section of grains in a unit gaseous mass, resulting in the opacity proportional to $\Sigma_{d} / a$. In our numerical calculations, the opacity is smoothly interpolated in the transition range where $a$ is comparable with $\lambda$.

\section{Numerical Results}

\subsection{Parameters and initial conditions}

Let us refer our first calculation as a standard case whose parameters and initial conditions are tabulated in Table 1. One of major uncertainties is in the time variation of the accretion rate $\dot{M}(t)$. In this study a simple step function is used under the assumption that the sun is formed from an interstellar cloud of $1 M_{\odot}$. With the initial masses of $0.7 M_{\odot}$ for the protosun and $0.057 M_{\odot}$ for the nebula as will be mentioned soon, the radial accretion lasts for $2.4 \times 10^{5}$ yrs with the rate of $1 \times 10^{-6} M_{\odot} /$ yrs. The same calculation but for an exponentially decaying $\dot{M}(t)$ with a characteristic time of $1 \times 10^{6}$ yrs has confirmed that the results do not differ very much from the standard case. As to the luminosity $L$, it would be appropriate to put $L$ to be $1 L_{\odot}$, the mean luminosity among $\mathrm{T}$ Tauri stars compiled by Beckwith et al. (1990). In fact $L$ is the sum of the intrinsic luminosity from the interior of the protosun and the accretion luminosity $G M_{c} \dot{M}_{c} / R_{c}$ ( $R_{c}$ being the radius of the protosun). We have computed the accretion luminosity with the calculated $M_{c}$ and $\dot{M}_{c}$ and with the mean $R_{c}$ among $\mathrm{T}$ Tauri stars $(=2.4$ solar radius)

Table 1. The parameters and initial conditions adopted for the standard case.

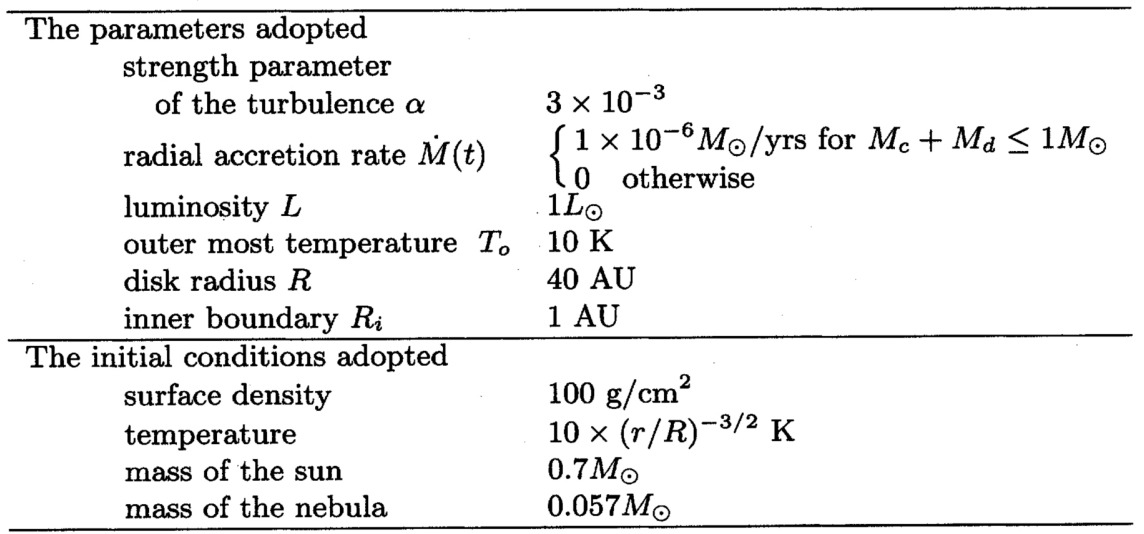


and obtained the time average of about $0.5 L_{\odot}$, showing the consistency of our choice of the value for $L$.

Our results are not very sensitive to the initial conditions. The initial mass distribution is readily masked by the accreting cloud gas after a few $\times 10^{5}$ yrs, comparable with the typical diffusion time. Also the initial temperature distribution is shown to be relaxed quickly because the temperature is essentially determined by the local and instantaneous balance of heating and cooling (see below about these points). In order to see how the results depend on the initial mass of the protosun, another calculation has been done for $M_{c}=0.5 M_{\odot}$ at $t=0$. Serious differences are not found except that the evolution takes longer time.

\subsection{Evolution during the accretion phase}

The structure of the nebula is shown in Fig. 1 for the accretion phase. During this stage, the accreting cloud gas is mostly deposited in the nebula because the accretion time is not long enough to carry the accreting gas to the protosun through diffusion. This is readily seen from the diffusion time defined as $t_{\text {diff }}=r^{2} / \nu=7 \times 10^{3} \times(r / 1 \mathrm{AU})^{2} /\left(\nu / 1 \times 10^{15} \mathrm{~cm}^{2} / \mathrm{sec}\right) \mathrm{yrs}$. For example, if we use $\nu=2 \times 10^{15} \mathrm{~cm}^{2} / \mathrm{sec}$ at $r=20 \mathrm{AU}$ and $t=1.0 \times 10^{5} \mathrm{yrs}, t_{\text {diff }}$ is about $1 \times 10^{6} \mathrm{yrs}$, longer than the accretion time of $2.4 \times 10^{5}$ yrs. At $2.4 \times 10^{5}$ yrs (just before the accretion ends), the
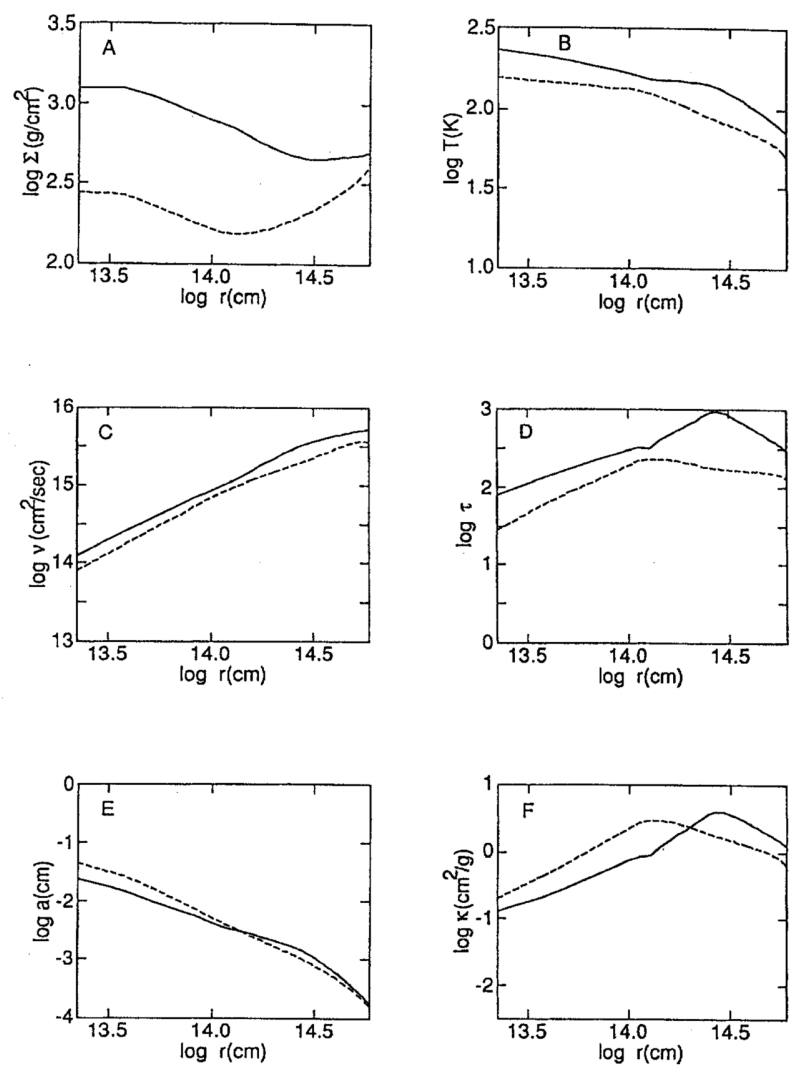

Fig. 1. The radial structure of the nebula during the accretion phase. The dashed and solid curves are for $1.0 \times 10^{5}$ yrs and $2.4 \times 10^{5} \mathrm{yrs}$, respectively. A: the surface density $\left(\mathrm{g} / \mathrm{cm}^{2}\right), \mathrm{B}$ : the temperature $(\mathrm{K}), \mathrm{C}:$ the turbulent viscosity $\left(\mathrm{cm}^{2} / \mathrm{sec}\right), \mathrm{D}$ : the optical depth, E: the mean radius of the grains $(\mathrm{cm})$, and $\mathrm{F}$ : the grain opacity $\left(\mathrm{cm}^{2} / \mathrm{sec}\right)$. 
nebula mass reaches $0.28 M_{\odot}$ while the mass increase of the protosun is merely $0.008 M_{\odot}$. At that time, the distribution of the surface density is approximately given as $1200(\mathrm{r} / 1 \mathrm{AU})^{-1 / 2} \mathrm{~g} / \mathrm{cm}^{2}$ within about $20 \mathrm{AU}$ and is nearly constant about $500 \mathrm{~g} / \mathrm{cm}^{2}$ outside $20 \mathrm{AU}$ (Fig. 1A).

The nebula is kept very opaque during this stage (Fig. 1D). With the increasing surface density, the increasing optical depth raises the temperature: for example, $240 \mathrm{~K}$ at $1 \mathrm{AU}$ and 70 $\mathrm{K}$ even at $40 \mathrm{AU}$ at $2.4 \times 10^{5} \mathrm{yrs}$. The temperature is found, at this time, to vary approximately as $r^{-0.3}$ inside about $10 \mathrm{AU}$, and as $r^{-1}$ beyond about $20 \mathrm{AU}$ (Fig. 1B). The different dependences are caused mainly by the opacity law: in the inner region the grains grow faster and go into the geometrical cross section regime while the Rayleigh limit still holds in the outer region owing to the slower growth and the addition of $1 \mu \mathrm{m}$ interstellar grains. The temperature is kept about $150 \mathrm{~K}$ in the intermediate region where the sublimation of solid ice reduces the opacity. Note that the detailed treatment of sublimation/recondensation guarantees the smoothness of the spatial distributions for all quantities, differently from most of the previous studies on the nebula structure where all solid ice is assumed to evaporate in the region where $T>$ a given critical temperature (Lin and Papaloizou, 1985; Cabot et al., 1987b). The temperature is indicated to be determined nearly by the local and instantaneous balance of heating and cooling in most region. The dominant heat sources are the solar irradiation and the dissipation (see Fig. 4A). In the standard case the solar irradiation exceeds the dissipative heating by a factor of a few or more. The vertical radiation loss almost balances with these heatings. The other terms in Eq. (6) is found to be important only in the innermost and outermost regions.

It is interesting to know whether or not the radial flow can become steady during the accretion phase. We have checked the mass flux $2 \pi r \Sigma v_{r}$. At $2.4 \times 10^{5} \mathrm{yrs}$, the inward flux is shown to decrease monotonically from $1 \times 10^{-6} M_{\odot} /$ yrs at $r=R$ (fixed by the outer boundary condition) to $1.1 \times 10^{-7} M_{\odot} /$ yrs at $r=R_{i}$ (in terms of velocity, it is almost constant to be $-35 \pm 10 \mathrm{~cm} / \mathrm{sec}$ ). This indicates that the steady flow is not necessarily attained during the accretion phase, which is obvious because the diffusion time is longer than the accretion time as seen above. Thus, the assumption of steady flow, usually made in the studies on accretion disk solar nebulae (Lin and Papaloizou, 1985; Cabot et al., 1987b; Mizuno, 1989), is applicable only to the cases of much larger viscosity or of much longer accretion time (see Case I and II in Subsection 3.4).

\subsection{Evolution during the post-accretion phase}

After the accretion ceases, the nebula loses its mass and the protosun acquires the mass, keeping the protosun/nebula system's mass constant. In Fig. 2, the grain radius, the opacity, the viscosity, and the radial flux of gas are plotted versus time for $5 \mathrm{AU}$ and $35 \mathrm{AU}$, chosen from the hot inner and cold outer regions, respectively. The spatial distributions of the same quantities as in Fig. 1 are shown in Fig. 3.

Let us see the grain's growth curves which, in general, include three effects: the collision, the transportation, and the variation of $\Sigma_{d}$. Two colliding grains are assumed in this study to merge into one, increasing the grain radius. The transportation usually reduces the grain radius, because the grain flux directs toward the protosun and because the grains are smaller in the outer region. The sublimation/recondensation is an example of the third effect.

The grain growth at $35 \mathrm{AU}$ gives us a typical example. During the accretion phase the injection of small interstellar grains at the outer boundary and the efficient transportation from the outer region suppress the growth of mean size. Indeed the advection term in Eq. (10) cancels, to the large extent, the collision term. Therefore, although the collision time, defined by $t_{c}=$ $2 /\left(n \sigma_{c} v_{c}\right)=0.15 t_{k}\left(\rho_{s} \Sigma a\right)^{1 / 2} / \Sigma_{d} /\left(v_{t} / c_{s}\right)^{3 / 2}\left(t_{k}\right.$ : the Keplerian period), is as short as $1 \times 10^{4} \mathrm{yrs}$ at $2.4 \times 10^{5} \mathrm{yrs}$, the mean grain radius is kept being of the order of $\mu \mathrm{m}$ during the accretion phase. This balance breaks when the accretion ceases. At this time, the nebula adjusts itself to the change of the outer boundary condition soon in the outer region so that the fluxes of the gas and the grains decrease (see Fig. 2C). Hence the collisional effect dominates the transportational 

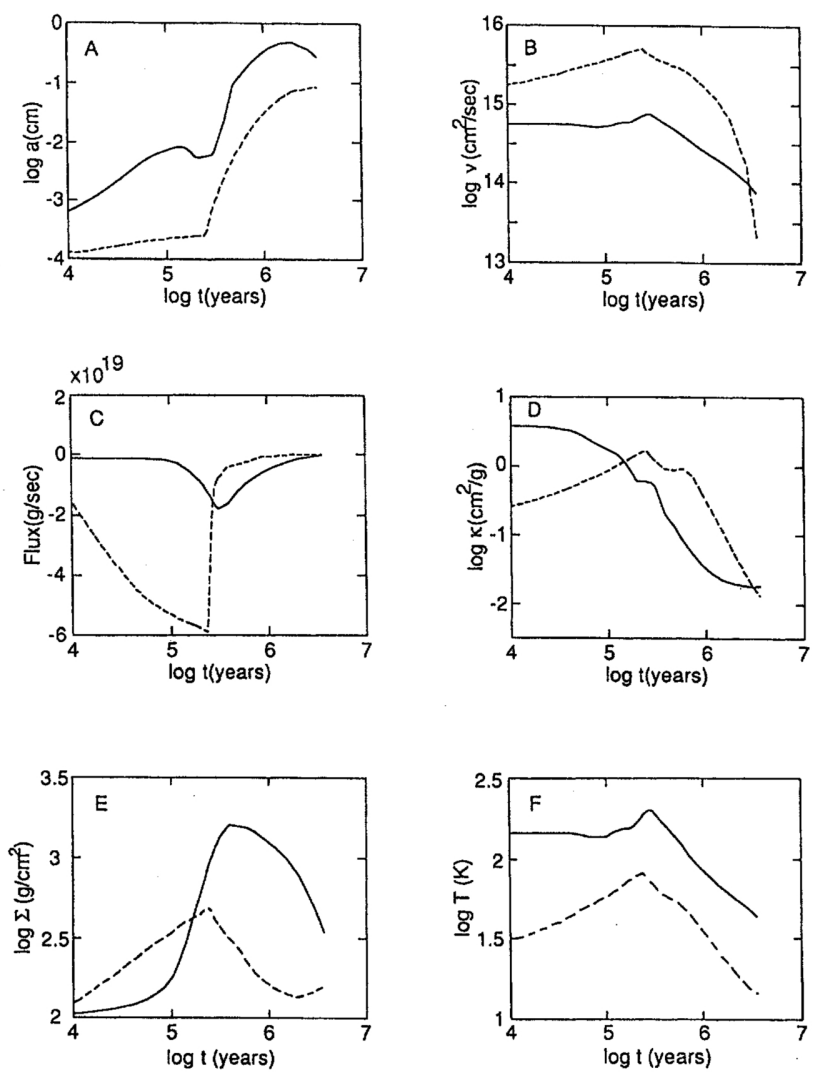

Fig. 2. Some quantities are plotted as a function of time. The solid and dashed curves are for $5 \mathrm{AU}$ and $35 \mathrm{AU}$, respectively. A: the mean radius of the grains $(\mathrm{cm}), \mathrm{B}$ : the turbulent viscosity $\left(\mathrm{cm}^{2} / \mathrm{sec}\right), \mathrm{C}$ : the gaseous flux $(\mathrm{g} / \mathrm{sec}), \mathrm{D}:$ the grain opacity $\left(\mathrm{cm}^{2} / \mathrm{g}\right)$, E: the surface density $\left(\mathrm{g} / \mathrm{cm}^{2}\right)$, and F: the temperature $(\mathrm{K})$. Notice from $\mathrm{C}$ that the termination of the accretion soon affects the structure in the outer region while the effect appears later in the inner region. The inward flux is decreasing rapidly in the late post-accretion phase in the whole nebula.

one. Thus the mean grain radius grows rapidly with $t_{c}=1 \times 10^{5} \mathrm{yrs}$ at $7.3 \times 10^{5} \mathrm{yrs}$. At the end of calculation $\left(t=3.6 \times 10^{6} \mathrm{yrs}\right)$, the radius is about $0.1 \mathrm{~cm}$. With the increasing radius, the decreasing collision rate is overcome by the effect of the transportation. Hence subsequently the grains no longer grow in the outer region and only change their position.

At $5 \mathrm{AU}$, the grains evolve in the same way as $35 \mathrm{AU}$ except that the sublimation/ recondensation occurs. When the temperature exceeds about $150 \mathrm{~K}$ at $1.6 \times 10^{5} \mathrm{yrs}$, the solid ice starts to evaporate and the grains begin to shrink. After the evaporation has completed at $2.2 \times 10^{5}$ yrs at $T=170 \mathrm{~K}$, the rock/metal grains grow again. When the grain flux decreases owing to the end of the accretion as in the region of $35 \mathrm{AU}$, the grains begin to grow quickly with $t_{c}=1 \times 10^{4}$ yrs. Meanwhile the temperature turns to decrease as stated below. Then the growth is further accelerated by the recondensation of ice vapor which happens to occur between $3.9 \times 10^{5}$ yrs and $4.8 \times 10^{5} \mathrm{yrs}$. The flow of smaller grains from the upper stream becomes noticeable after about $1 \times 10^{6}$ yrs. Note that the sublimation occurs when the grain radius is about $10^{-2} \mathrm{~cm}$ at $5 \mathrm{AU}$. This means that the equilibrium between solid ice and ice vapor indeed holds as assumed in Subsection 2.3. 

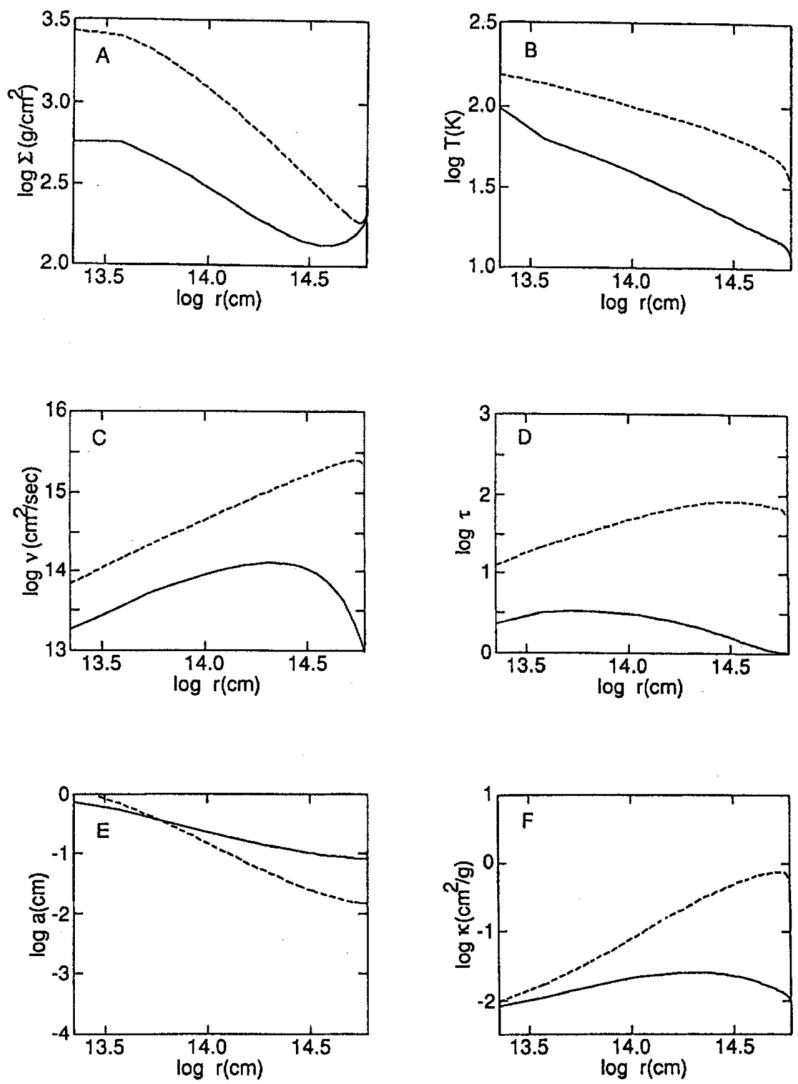

Fig. 3. The same as Fig. 1, but for the post-accretion phase. The dashed and solid curves are for $7.4 \times 10^{5}$ yrs and $3.6 \times 10^{6} \mathrm{yrs}$, respectively.

During the post accretion stage, the growing grains go beyond the Rayleigh limit regime even in the outer region. Decreasing as the grains grow, the opacity reduces the optical depth cooperatingly with the decreasing surface density. The small optical depth plays essential roles for the subsequent nebula evolution as pointed by RP. The first consequence is in lowering the temperature. There are two reasons for this. The first reason is the more efficient radiative loss with the smaller optical depth. The second reason is the reduction of dissipative heating, which is caused by the decreasing viscosity as to be mentioned soon below. In Fig. 4 we compare three heating sources of dissipation, solar irradiation, and cosmic ray for two epochs chosen from the accretion phase and the post-accretion phase. From the figure, the viscous dissipation, less than the other energy sources by many orders of magnitude, is no more the major source to maintain the temperature in the last post-accretion stage. This is true for a case of $\alpha$ as large as $10^{-2}$. Thus the temperature decreases; for example, below $150 \mathrm{~K}$ in the whole nebula by $7.4 \times 10^{5} \mathrm{yrs}$ (Fig. 2F and Fig. 3B) so that all the ice is in solid afterward. At $3.6 \times 10^{6} \mathrm{yrs}$, the temperature distribution is found to be given approximately by $100 \times(r / 1 \mathrm{AU})^{-1 / 2} \mathrm{~K}$.

The second consequence of small optical depth is the decay of convection, and therefore, the decrease of viscosity (Fig. 2B) which affects the surface density (Fig. 2E and Fig. 3A). After about $4 \times 10^{5} \mathrm{yrs}$, the surface density continues to decrease in the whole nebula owing to the gas outflow from the inner edge and owing to the absence of the accretion. The decreasing viscosity 

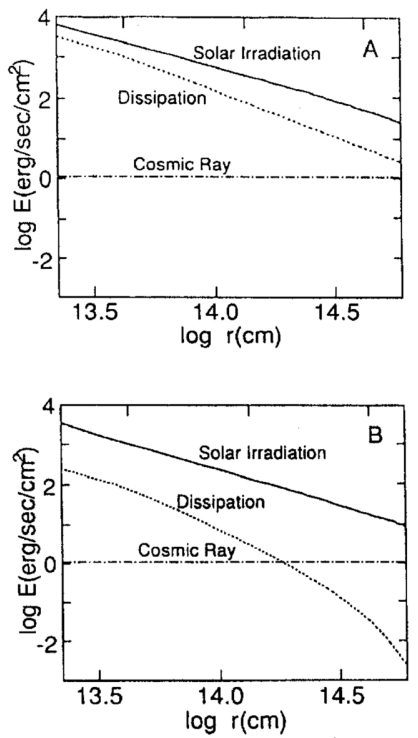

Fig. 4. The comparison of heating sources. Three heating sources are compared at two epochs for the standard case. A: $2.4 \times 10^{5}$ yrs, B: $3.6 \times 10^{6}$ yrs.

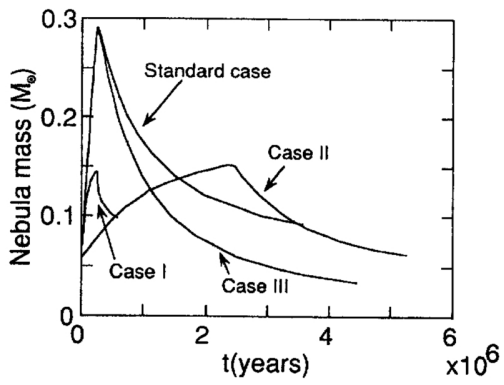

Fig. 5. The nebula mass is plotted versus time for the four cases for which the computations have been made. In spite of the difference in the adopted values of the parameters, the final nebula mass varies within a rather small range.

reduces the inward mass flux as shown in Fig. $2 \mathrm{C}$ by a factor of about a few $\times 10$ times as small as the maximum. (The speed of radial drift is on the order of $1 \mathrm{~cm} / \mathrm{sec}$ in the entire nebula.) And the surface density changes less and less in time. At $3.6 \times 10^{6}$ years, the time scale $|\Sigma /(d \Sigma / d t)|$ is about $2 \times 10^{6}$ years at $5 \mathrm{AU}$ and about $8 \times 10^{6}$ years at $35 \mathrm{AU}$. The time scale will become longer rapidly because of the sharp drop of viscosity (Fig. 2B). This situation is also obviously seen in Fig. 5 which shows that the nebula mass $M_{d}$ approaches asymptotically to about 0.1 solar mass as time passes. The life time of the nebula, defined $\left|M_{d} / \dot{M}_{d}\right|$, is the order of $1 \times 10^{7}$ years at $3.6 \times 10^{6}$ years. Thus the weakly convecting nebula is being remained.

At $3.6 \times 10^{6} \mathrm{yrs}$, the surface density is given approximately as $600 \times(\mathrm{r} / 1 \mathrm{AU})^{-3 / 4} \mathrm{~g} / \mathrm{cm}^{2}$ except the outermost region where the density inversion is seen. It should be noted that this distribution is a direct consequence of grain growth as to be mentioned in Subsection 3.5. By 
the way, this inversion is caused by the following reason. In the region with $r>20 \mathrm{AU}, \tau$ close to unity makes $\nu$ decrease rapidly with $r$, resulting in positive $v_{r}$ as seen from Eq. (5). Thus the disk should spread. However our numerical boundary condtion in Subsection 2.4 forbids the gas to flow out from the outer boundary. Therefore the gas piles up there to make the inversion of the surface density.

These numerical values have uncertainties because of many crude treatments involved in our study. However the computation for the standard case at least implies the following three points. First, as the thermal convection weakens, the nebula can be left naturally within a framework of star formation through an accretion disk. Second, the nebula mass seems to be consistent with that assumed a priori in the conventional studies of the planetary formation. Third, as long as we are concerned with the accretion disk model, the nebula can remain long enough for the terrestrial planets to be formed (Ohtsuki et al., 1988).

\subsection{Sensitivity of the nebula mass to parameters}

Hereafter we concentrate on the nebula mass and see how it depends on parameters. The reason to focus on the nebula mass is not only that our treatment clearly prefers the discussion of the integrated quantities to the detailed structure, but also that our calculation can give some implication on the remaining nebula mass which has been given a priori so far in the theory of planetary formation. For this purpose, we have performed three additional calculations. In each case only one parameter is changed from the standard case: $\alpha=3 \times 10^{-2}$ in Case I, $\dot{M}(t)=1 \times 10^{-7} M_{\odot} /$ yr for $M_{c}+M_{d}<1 M_{\odot}$ in Case II, and no collisional growth of grains in Case III. Case III is considered from the claim of Weidenschilling (1984) that the collision velocity by Völk et al. is overestimated because the inner scale of turbulent eddy was set to be zero instead of finite. Hence we have tried to examine another extreme in Case III. In addition, we have done two more calculations for $q=0.1$ and 0.4 since we found the importance of the energy sources besides the dissipation at the final stage as mentioned above.

The nebula mass is plotted versus time in Fig. 5 for Case I, II, and III. The most important conclusion to be deduced from Fig. 5 is that the remaining mass of the nebula is not very sensitive to $\alpha, \dot{M}(t)$, and collision velocity if they vary within the range adopted here. In Table 2 the nebula mass of the model at the end of computation for each case is summarized as well as the time scale of $\left|M_{d} / \dot{M}_{d}\right|$. From the computations for the cases of $q=0.1$ and 0.4 we have found that the disk mass for these cases evolve with the small deviation of less than about thirty percent from the standard case (hence omitted from Fig. 5 and Table 2). For example, we have the final disk masses of 0.12 solar mass and 0.08 solar mass for $q=0.1$ and 0.4 , respectively. Thus our numerical ambiguity for $q$ does not affect the disk mass if it is within a factor of two. These nebula masses are confined in the narrow range from about 0.03 solar mass about 0.1 solar mass. Interestingly this range nearly covers the assumed nebula mass in the conventional theories of planetary formation. Let us regard the time scale as the life time of the nebula. Then, unless other processes such as solar wind work efficiently, the nebula seems to survive long enough for the terrestrial planets to be formed (Ohtsuki et al., 1988). Notice that, in any case, the period

Table 2. The nebula mass at the end of the computation (in $M_{\odot}$ ) and its life time (in yrs).

\begin{tabular}{lcc}
\hline & nebula mass & life time \\
\hline Standard case & 0.09 & $1.3 \times 10^{7}$ \\
Case I & 0.10 & $2.7 \times 10^{6}$ \\
Case II & 0.06 & $8.2 \times 10^{6}$ \\
Case III & 0.03 & $5.0 \times 10^{6}$ \\
\hline
\end{tabular}


exists when the nebula mass exceeds 0.1 solar mass. Hence the nebula may be gravitationally unstable during this time. We will comment on this point in Section 4.

Let us see how each parameter affects the nebula mass. The influence of the greater $\alpha$ is seen in two points (Case I). First, the maximum nebula mass is about half of that for the standard case because of the larger viscosity. Second, the greater $\alpha$ shortens the time during which the nebula remains, about ten times shorter than that for the standard case. This is due to the fast grain growth and, therefore, the fast decrease in viscosity. The smaller $\dot{M}(t)$ or the longer accretion time in Case II gives enough time for the gas to drift over the nebula radius, which lowers the maximum mass of the nebula to about the same as in Case I. The steady flow is found to be almost achieved by the end of the accretion in Cases I and II. ${ }^{1}$

In Case III the nebula mass in the accretion phase is practically the same as that for the standard case because the effect of grain growth is negligible also in the standard case outside about $10 \mathrm{AU}$. The discrepancy is seen in the post-accretion phase. The temperature is always higher than the standard case because of the greater optical thickness due to the small grains. Therefore the viscosity is greater and the faster drift flow makes the nebula mass smaller.

\subsection{Comparison with Ruden and Pollack (1991)}

Here we compare our results with those of RP. As they pointed out, the remaining nebula mass would be expressed as

$$
M_{d}=\int_{R_{i}}^{R} \frac{2}{\kappa} 2 \pi r d r,
$$

where the nebula is assumed to be left with $\tau$ nearly equal to unity. Thus the opacity is a decisive factor. Adopting the opacity for interstellar grains with solar abundance, they enable to indicate an analytic form of the remaining nebula mass by using the temperature obtained from the viscous heating and the radiative loss. On the other hand, the opacity calculation is difficult in our case because of the following reaons. One is the time-dependent size of grains as already described above. Another is the grain abundance which is not always solar if the grains are $\mathrm{mm}-\mathrm{cm}$ in size (see Fig. 6 for example). The grains of this size continue to drift inward owing to the gas drag even if the gaseous radial motion is ceasing at this time. This causes the deviation from the solar abundance. Therefore the remaining mass of the nabula is, in our case, actually an evolutional result which combines several effects.

Our remaining mass is even smaller than their results. For example, 0.09 solar mass is left within $40 \mathrm{AU}$ in our standard case while their Eq. (25) shows the remnant mass to be about 0.17 solar mass within the same region for $\alpha=3 \times 10^{-3}$. Their remnant mass is also larger than that for our Case III (no collisional growth). The reason is their neglect of the solar irradiation and the cosmic ray as energy sources. In fact, as mentioned in Subsection 3.3 and seen in Fig. 4, these heat souraces dominate the dissipation at the final stage when the viscosity decreases. As a result their temperature decreases to less than several Kelvin in the wide region of $r>1$ AU in the final stage. Hence the opacity becomes small, quenching the drift flow earlier. Thus the remnant mass is larger than our Case III. This implies that, in order to obtain the remaining mass, the energy sources at the final stage are important.

Another difference is the $r$-dependence of the surface density distribution of the remaining nebula. In Fig. 6 the surface densities of gas and solid grains are plotted for the remaining nebulae for our standard case and their critical surface density (using $\alpha=3 \times 10^{-3}$ in Eq. (24) of RP). For comparison, Hayashi's solar nebula model is added to the figure. The surface density distribution of the remaining nebula has a different tendency, depending to the adopted opacity.

\footnotetext{
${ }^{1}$ Under some reasonable assumptions, we have found a scaling law for the nebula structure which holds for the accretion phase. The scaling law shows that $\Sigma$ and $t$ are scaled with both $\alpha$ and $\dot{M}$. We do not continue this discussion more for the brevity of this paper.
} 


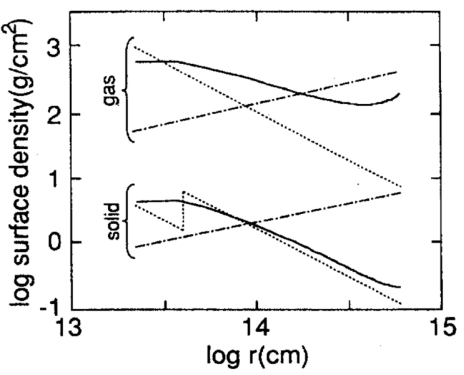

Fig. 6. The distribtions of the surface densities of gas and solid is plotted for the final model (solid line). For comparison, the same are shown for Ruden and Pollack's remnant (dash-dotted line) and Hayashi's solar nebula model (dotted line).

RP obtain the surface density proportional to $r^{3 / 5}$ from the opacity in the Rayleigh limit regime. However in our case the opacity depends on the grain radius and abundance. Our surface density is found to be nearly proportional to $r^{-3 / 4}$, which has a trend similar to Hayashi's solar nebula. This comparison indicates that the grains' behavior during the nebular evolution is significant on the structure of the remaining quiet nebula, in which the grains begin to sediment toward the mid-plane to form planetesimals.

\section{Conclusions}

We have considered the evolutionary behavior of the nebula. The evolution is inevitably non-steady because of the following main factors: the time dependent accretion of interstellar cloud and the collisional growth of grains. We should not deduce quantitative conclusions from our present calculations owing to many approximations and the limited range of parameters. However the results are considered to indicate the qualitative evolutionary sequence.

As first pointed out by RP, the non-turbulent nebula remains after the termination of the accretion. In our case this is caused by the grain growth in the nebula. The remaining mass of the nebula seems to be in the narrow range around 0.05 solar mass, even smaller than RP's estimate. This remaining nebula has the mass enough to form planets and has the life time long enough for the terrestrial planets to accumulate. Interestingly the parameter $\alpha$ and the accretion rate $\dot{M}(t)$ have only a weak influence on the remaining mass as long as they change within a factor of ten from the standard values.

The comparison with RP indicates the followings. First, the grains' behavior is significant for the remnant mass and its spatial distribution. The grains grow in the convection, the opacity drops, the convection weakens, the viscosity decreases, and finally the nebula is left. If the grains grow beyond the wavelength, their size has a direct connection to the surface density distribution. Second, the energy sources such as the solar irradiation and the cosmic ray are more important than the viscous dissipation at the final stage in determining the remnant mass with an accuracy within a factor of a few. In fact our remaining mass is smaller than RP's result by a factor of about two. This means that for a more precise estimate of the remnant mass, the energy equation needs more careful treatment for the solution in the future, considering the local curvature of the nebula surface, the shadow effect, and so on.

Until now some difficulties have been pointed out in the convective accretion disk nebula. One of them is that, in the turbulent nebula, the grains cannot settle to the central plane (Weidenschilling, 1984; Battaglia, 1987; Mizuno, 1989). However this difficulty can be avoided naturally in the present scenario because the convection weakens as the grains grow. In our computation, 
the grain size becomes as large as $\mathrm{mm}-\mathrm{cm}$ by the termination of convection. Because of the short time scale of sedimentation for these large grains, it can be considered that the grains sediment in fact instantly when the convection ends. In this way the planetesimals are formed. Hence the initial mass distribution of planetesimals, which has been an ad hoc assumption in the theories of planetary formation, can be obtained as a outcome of the non-steady evolution of the nebula.

We conclude this study by pointing out the further problems contained in this study. First, the vertical structure of the nebula is assumed to be uniform, which prevents us from using the exact criterion for thermal convection. In relation to this, we implicitly overlook an additional effect of solar irradiation other than heating, i.e., the effect that it tends to suppress the convection as pointed out by Watanabe et al. (1990). Second, the gravitational instability is met in our nonsteady case: $1<\Sigma / \Sigma_{\text {crit }}<3$ (where $\Sigma_{\text {crit }}=\Omega c_{s} / \pi G$ ) beyond about 30 AU at $2.4 \times 10^{5}$ yrs in the standard case, for example. This instability is also found in the steady accretion model (Cabot et $a l ., 1987 \mathrm{~b}$ ) and in the vertical accretion model (Nakamoto and Nakagawa, in preparation). It is necessary to investigate what the onset of this instability produces: giant protoplanet (Cameron, 1978) or another turbulent state (Lin and Pringle, 1990). Third, we substitute mean grains for the mass spectrum of grains. Whether or not this treatment is a good approximation of the actual mass spectrum is necessary to confirm in the future.

Although these problems are left to be solved in the future, the conclusion of this paper is significant from the following point of view. That is, we have shown that the nebula disk around the protosun (and T Tauri stars) evolves from the active and turbulent state to the quiet state owing to the growth of grains. This evolution is doomed by the mechanism involved in the nebula itself. Thus the earliest stage of the solar system is connected with the star formation.

This work was supported partly by the Gant-in-Aid for General Scientific Research of the Japanese Ministry of Education, Science, and Culture (No. 02452062).

Appendix: Approximate evaluation of the effect of erosion on the grain growth and opacity

As described in Section 2, we approximate the size distribution of grains as that with two sizes in order to roughly evaluate the effect of erosion on collisions. One is the grains with mass $M$, radius $a_{M}$ and density $\rho(M)$. These grains grow by collisions although a part of colliding grains erode. Another is the grains with mass $m_{0}$, radius $a_{m_{0}}$ (assumed to be $1 \mu \mathrm{m}$ ) and density $\rho\left(m_{0}\right)$. The latter grains are the smallest grains returned by the erosion. The density $\rho\left(m_{0}\right)$ would be determined by 1 ) the supply of grains $m_{0}$ owing to the ersion by collisions between grains $M, 2)$ the loss of grains $m_{0}$ owing to the growth by collisions between the grains $m_{0}$, and 3 ) the loss of grains $m_{0}$ owing to the coagulation by collisions between the grains $M$ and $m_{0}$. The erosion on collisions between $M$ and $m_{0}$ is negligible. Then we have a following equation.

$$
2 m_{0} \frac{1}{2}\left\{\frac{\rho\left(m_{0}\right)}{m_{0}}\right\}^{2}\left(\sigma_{c} v_{c}\right)_{m_{0} m_{0}}+m_{0} \frac{\rho\left(m_{0}\right)}{m_{0}} \frac{\rho(M)}{M}\left(\sigma_{c} v_{c}\right)_{m_{0} M}=m_{e x} \frac{1}{2}\left\{\frac{\rho(M)}{M}\right\}^{2}\left(\sigma_{c} v_{c}\right)_{M M}
$$

where $m_{e x}$ is the eroded mass on each collision between the grains $M$ and is given by

$$
m_{e x}=0.1 \frac{\rho_{s}}{E_{s}} \frac{M \cdot\left(v_{c}\right)_{M M}^{2}}{8}
$$

following Battaglia's treatment (1987). In this equation $E_{s}$ is the impact strength. We use the collision velocity made to reproduce the results of Völk et al. (1980), that is,

$$
v_{c}=\alpha c_{s} \frac{1}{\sqrt{2}}\left\{\sqrt{\frac{\tau_{f 1}}{\tau_{k 0}}}+\sqrt{\frac{\tau_{f 2}}{\tau_{k 0}}}\right\},
$$


where $\tau_{f}$ and $\tau_{k 0}$ are the friction time of a collising grain and the life time of the largest turbulent eddy. In the case of $M \gg m_{0}$ or $a_{M} \gg a_{m_{0}}$, the above equation becomes

$$
x^{2}+\frac{1}{8}\left(\frac{a_{m_{0}}}{a_{M}}\right)^{1 / 2} x-\frac{1}{2} \frac{m_{e x}}{M}\left(\frac{a_{m_{0}}}{a_{M}}\right)^{1 / 2}=0,
$$

where $x=\rho\left(m_{0}\right) / \rho(M)$. The solution of this equation is shown in the table below for the typical values for parameters: $a_{m_{0}}=1 \mu \mathrm{m}, a_{M}=1 \mathrm{~cm}, E_{s}=5 \times 10^{4} \mathrm{erg} / \mathrm{cm}^{3}, T=200 \mathrm{~K}$ and $\Sigma=1000$ $\mathrm{g} / \mathrm{cm}^{2}$. From the table we find that the eroded mass is at most a percent even for $\alpha=0.1$. For $a_{M}<1 \mathrm{~cm}$, the ratio should be smaller than those in the table. Thus the erosion does not affect the grain growth very much as long as $a_{M}<1 \mathrm{~cm}$.

Next we consider the effect on the grain opacity. When $a_{M}$ is smaller than the wavelength $\lambda$, the grains $m_{0}$ and $M$ are in the Rayleigh limit regime where the grain opacity is proportional to mass density. Hence, from the result in the previous paragraph, the influence of erosion on opacity can be ignored if $a_{M}<\lambda$. However where the grains $M$ enter into the regime of $a_{M}>\lambda$, the opacity due to grains $M$ decreases as they grow. Hence the grains $m_{0}$ can affect the total opactiy even though their mass density is small. To estimate this, we use the following expression for the ratio of opacity contributed from grains $m_{0}$ to that from grains $M$

$$
\frac{\kappa\left(m_{0}\right)}{\kappa(M)}=\frac{2 \rho_{s} a_{M} \kappa_{\text {solar }}}{3 A} \frac{\rho\left(m_{0}\right)}{\rho(M)}
$$

where $A=0.016$ and $\kappa_{\text {solar }}$ is the interstellar grain opacity for solar composition. This ratio is shown also in the table below for the same parameters. For $\alpha=0.1$, the eroded grains dominate the growing grains in opacity, which coincides with the results of Weidenschilling (1984). However the contribution from the eroded grains is less than the order of 0.1 for the interesting range of $\alpha$ from $10^{-4}$ to $10^{-2}$. This result indicates that the erosion is unlikely to affect on both grain mass and grain opacity in the solar nebula at least if the grain size is smaller than $1 \mathrm{~cm}$. Therefore we expect our approximation of grain growth in this paper to be rather good.

$$
\begin{array}{ccccc}
\alpha & 10^{-4} & 10^{-3} & 10^{-2} & 10^{-1} \\
\rho\left(m_{0}\right) / \rho(M) & 2 \times 10^{-10} & 2 \times 10^{-7} & 4 \times 10^{-4} & 2 \times 10^{-2} \\
\kappa\left(m_{0}\right) / \kappa(M) & 1 \times 10^{-7} & 1 \times 10^{-4} & 3 \times 10^{-1} & 1 \times 10^{1}
\end{array}
$$

\section{REFERENCES}

Adams, F. C., C. J. Lada, and F. H. Shu, The disk of T Tauri stars with flat infrared spectra, Astrophys. J., 326, 865-883, 1988.

Allen, C. W., Astrophysical Quantities, The Athlone Press, 1973.

Battaglia, A., Growth and sedimentation of dust grains in the primitive solar nebula, Ph.D. Dissertation, Graduate Faculty in Physics, The City University of New York, 1987.

Beckwith, S. V. W., A. I. Sargent, R. S. Chini, and R. Gusten, A Survey for circumstellar disks around young stellar objects, Astron. J., 99, 924-945, 1990.

Bertout, C., T Tauri stars: Wild as dust, Ann. Rev. Astron. Astrophys., 27, 351-395, 1989.

Cabot, W., V. M. Canuto, O. Hubickyj, and J. B. Pollack, The role of turbulent convection in the primitive solar nebula I: Theory, Icarus, 69, 387-422, 1987a.

Cabot, W., V. M. Canuto, O. Hubickyj, and J. B. Pollack, The role of turbulent convection in the primitive solar nebula II: Results, Icarus, 69, 423-457, $1987 \mathrm{~b}$.

Cameron, A. G. W., Physics of the primitive solar accretion disk, Moon and Planets, 18, 5-40, 1978.

Cameron, A. G. W. and M. B. Fegley, Nucleation and condensation in the primitive solar nebula, Icarus, 52, 1-13, 1982.

Goldreich, P. and W. R. Ward, The formation of planetesimals, Astrophys. J., 150, 1051-1061, 1973.

Hayashi, C., Structure of the solar nebula, growth and decay of magnetic fields and effects of magnetic and turbulent viscosities on the nebula, Prog. Theor. Phys. Suppl., 70, 35-53, 1981. 
Hayashi, C., K. Nakazawa, and Y. Nakagawa, Formation of the solar system, in Protostars and Planets, Vol. II, edited by D. C. Black and M. S. Matthews, 1100 pp., Univ. of Arizona Press, 1985.

Kenyon, S. J. and L. Hartmann, Spectral energy distribution of T Tauri stars: Disk flaring and limits on accretion, Astrophys. J., 323, 714-733, 1987.

Lin, D. N. C. and J. Papaloizou, On the structure and evolution of the primordial solar nebula, Mon. Not. R. Astron. Soc., 191, 37-48, 1980.

Lin, D. N. C. and J. Papaloizou, On the dynamical origin of the solar system, in Protostars and Planets, Vol. II, pp. 981-1072, Univ. of Arizona Press, 1985.

Lin, D. N. C. and J. E. Pringle, The formation and initial evolution of protostellar disks, Astrophys. J., 358, 515-524, 1990.

Meakin, P. and B. Donn, Aerodynamic properties of fractal grains: Implications for the primordial solar nebula, Astrophys. J., 329, L39-L41, 1988.

Mizuno, H., Grain growth in the turbulent accretion disk solar nebula, Icarus, 80, 189-201, 1989.

Mizuno, H., W. Markiewicz, and H. J. Völk, Grain growth in proto-planetary accretion disks, Astron. Astrophys., 195, 183-192, 1988.

Morfill, G. E., Some cosmochemical consequences of a turbulent protoplanetary cloud, Icarus, 53, 41-54, 1983.

Morfill, G. E., Protoplanetary accretion disks with coagulation and evaporation, Icarus, 75, 371-379, 1988.

Morfill, G. E. and H. J. Völk, Transport of dust and vapor and chemical fractionation in the early protostar cloud, Astrophys. J., 287, 371-395, 1984.

Nakagawa, Y., K. Nakazawa, and C. Hayashi, Growth and sedimentation of dust grains in the primordial solar nebula, Icarus, 45, 517-528, 1981.

Nakamoto, Y. and Y. Nakagawa, submitted to Astrophys. J., 1992.

Ohtsuki, K., Y. Nakagawa, and K. Nakazawa, Growth of the Earth in nebular gas, Icarus, 83, 552-565, 1988.

Pollack, J. B., C. P. McKay, and B. M. Christofferson, A calculation of the Rosseland mean opacity of dust grains in primordial solar system nebula, Icarus, 64, 471-492, 1985.

Ruden, S. P. and J. B. Pollack, The dynamical evolution of the protostar nebula, Astrophys. J., 375, 740-760, 1991.

Safronov, V. S., Evolution of the protoplanetary cloud and formation of the earth and the planets, Israel Program for Scientific Translations, 1972.

Safronov, V. S. and T. V. Ruzmaikina, Formation of the solar nebula and the planets, in Protostars and Planets, Vol. II, pp. 959-980, Univ. of Arizona Press, 1985.

Tscharnuter, W. M., Numerical studies of cloud collapse, in The Physics of Star Formation and Early Stellar Evolution, edited by C. J. Lada and N. D. Kylafis, 411 pp., Kluwer, 1991.

Völk, H. J., F. C. Jones, G. E. Morfill, and S. Röser, Collisions between grains in a turbulent gas, Astron. Astrophys, 85, 316-325, 1980.

Watanabe, S., Y. Nakagawa, and K. Nakazawa, Cooling and quasi-static contraction of the primitive solar nebula after gas accretion, Astrophys. J., 358, 282-292, 1990.

Weidenschilling, S. J., Evolution of grains in a turbulent solar nebula, Icarus, 60, 553-567, 1984. 